\title{
Differences in fully differential cross section of single top quark production and its subsequent decay process depending on var- ious anomalous Wtb couplings.
}

\author{
Edward Boos ${ }^{1, \star}$ and Viacheslav Bunichev ${ }^{1, \star \star}$ \\ ${ }^{1}$ Skobeltsyn Institute of Nuclear Physics, Lomonosov Moscow State University Leninskie Gory, 119991, \\ Moscow, Russia
}

\begin{abstract}
The fully differential cross sections of t-channel production of a single top quark with its subsequent decay are compared for the case of contributions involving anomalous Wtb couplings. It is shown that the most noticeable differences, for cases corresponding to different anomalous couplings, appear in the shape of two-dimensional distributions, where one of the variables is the energy of a charged lepton and the other is one of the t-quark spin orientation angles.
\end{abstract}

\section{Introduction}

Single top quark production and decay processes play a very important role, since they make it possible to measure the parameters of the Wtb vertex with high accuracy, as well as to study the polarization properties of the t-quark and its decay products. This allows you to find possible deviations from the predictions of the Standard Model [1-6]. If new physics exists on a large scale, then its contribution can be taken into account using operators of dimension six [7], which are invariant with respect to the gauge group of the Standard Model. The number of linearly independent operators is limited and among them one can construct a basis [8]. Some of the operators from this basis set can contribute to the processes of single top production [9-13]. Corrections to the Wtb vertex coming from the new physics can be parametrized by the Wilson coefficients corresponding to these operators. An effective Lagrangian including such corrections can be written as [14]:

$$
\mathcal{L}=-\frac{g}{\sqrt{2}} \bar{b} \gamma^{\mu}\left(f_{L V} P_{L}+f_{R V} P_{R}\right) t W_{\mu}^{-}-\frac{g}{\sqrt{2}} \bar{b} \frac{i \sigma^{\mu \nu}}{2 M_{W}}\left(f_{L T} P_{L}+f_{R T} P_{R}\right) t W_{\mu \nu}^{-}+\text {h.c. }
$$

where $M_{W}$ is the W-boson mass, $P_{L, R}=\left(1 \mp \gamma_{5}\right) / 2$ is the left(right)-handed projection operator, $W_{\mu \nu}^{-}=\partial_{\mu} W_{v}^{-}-\partial_{\nu} W_{\mu}^{-}, g$ is the weak isospin gauge coupling, parameters $f_{L V(T)}$ and $f_{R V(T)}$ are the dimensionless coefficients that parametrize strengths of the left-vector (tensor) and the right-vector (tensor) structures in the Lagrangian.

The couplings in the Lagrangian (1) are related in the following way to the corresponding Wilson coefficients:

\footnotetext{
$\star$ e-mail: boos@theory.sinp.msu.ru

$\star \star$ e-mail: bunichev@ theory.sinp.msu.ru
} 


$$
f_{L V}=V_{t b}+C_{\phi q}^{(3,33)} \frac{v^{2}}{\Lambda^{2}}, \quad f_{R V}=\frac{1}{2} C_{\phi u d}^{(33)} \frac{v^{2}}{\Lambda^{2}}, \quad f_{L T}=\sqrt{2} C_{d W}^{(33)} \frac{v^{2}}{\Lambda^{2}}, \quad f_{R T}=\sqrt{2} C_{u W}^{(33)} \frac{v^{2}}{\Lambda^{2}}
$$

The dominant process of single top production at the LHC collider is the t-channel process shown in the (Fig. 1). It is known that in the framework of the SM in a t-channel process $u b \rightarrow t d$, t-quark is produced highly polarized due to the $(\mathrm{V}-\mathrm{A})$ interaction. In the $\mathrm{SM}$, the positively charged lepton from the top quark decay in its rest frame tends to follow the top quark spin direction [16, 17]. In the t- and s-channel single top production the direction of the top quark spin in the top rest frame is highly correlated with the d-quark momentum for the t-channel (outgoing light jet) and anti d-quark momentum (incoming parton corresponding to the beam axis) for the s-channel $[18,19]$. The direction of preferred spin configuration in the single top production in presence of anomalous couplings is changed insignificantly comparing to SM due to smallness of the anomalous couplings. So one can chose the direction related to mentioned d-quark momentum as a top spin quantization axis (Fig. 2) and to make sure that this will be a preferred spin direction of top quark in its rest frame similar to SM [20]. For calculations we use coordinate system that shown in (Fig. 2). Here the angle $\theta$ is the angle between the charged lepton momentum and the direction of the top quark spin quantization axis, $\phi$ is the angle in the plane perpendicular to the lepton momenta counted from the decay plain formed by the top quark decay products, In [20] we obtained analytical expressions for the differential cross sections of the full process of production and decay of the t-quark $\left(u b \rightarrow d, b, v, e^{+}\right)$in its rest frame as a function of the energy of a charged lepton and two angles of orientation of the quantization axis of the t-quark spin. The expression is presented in the most general form for the case of real and imaginary vector and tensor anomalous Wtb couplings. To make deviations from the SM more pronounce we subtract the SM contribution. To correctly compare the shapes of different distributions, we normalized them to the value of the full integral. As follows from the [20] the leading terms up to second power on anomalous couplings have the form:

$$
\begin{aligned}
\frac{1}{I_{n o r m}} \frac{d\left(\sigma-\sigma_{S M}\right)_{u b \rightarrow d b v e^{+}}}{d \epsilon \cdot d \cos \theta \cdot d \phi} & =\frac{1}{\sigma_{u b \rightarrow t d} \Gamma_{t \rightarrow b v e^{+}}} \cdot\left[\left(\sigma_{S M}\right)_{u b \rightarrow t d} \cdot \frac{d\left(\Gamma-\Gamma_{S M}\right)_{t \rightarrow b v e^{+}}}{d \epsilon \cdot d \cos \theta \cdot d \phi}\right. \\
& +\left(\sigma_{u b \rightarrow t d}-r_{\Gamma} \cdot\left(\sigma_{S M}\right)_{u b \rightarrow t d}\right) \cdot \frac{d\left(\Gamma_{S M}\right)_{t \rightarrow b v e^{+}}}{d \epsilon \cdot d \cos \theta \cdot d \phi} \\
& +\left(\sigma_{R T}\right)_{u b \rightarrow t d} \cdot \frac{d\left(\Gamma_{R T}\right)_{t \rightarrow b v e^{+}}}{d \epsilon \cdot d \cos \theta \cdot d \phi} \\
& \left.-\left(\sigma_{R}\right)_{u b \rightarrow t d} \cdot V_{t b}^{2} \cdot \frac{\alpha^{2} \cdot m_{t}^{3}}{64 \cdot \pi \cdot \sin ^{4} \Theta_{W} \cdot \Gamma_{W} \cdot m_{W}} \cdot(1-\epsilon) \cdot \epsilon \cdot \cos \theta\right]
\end{aligned}
$$

where $\sigma_{u b \rightarrow t d}$ is the cross section of the unpolarized t-quark production and $\left(\sigma_{R}\right)_{u b \rightarrow t d}$ is a part of this cross section that proportional $\left|f_{R V}\right|^{2}$ and $\left|f_{R T}\right|^{2},\left(\sigma_{R T}\right)_{u b \rightarrow t d}$ is a part that proportional $V_{t b} \cdot R e f_{R T}$, $\left(\Gamma_{R T}\right)_{t \rightarrow b v e^{+}}$is a part of top partial width that proportional $V_{t b} \cdot \operatorname{Re} f_{R T}$ and $V_{t b} \cdot \operatorname{Im} f_{R T},\left(\sigma_{S M}\right)_{u b \rightarrow t d}$ is the SM cross section of the unpolarized t-quark production, $r_{\Gamma}=\frac{\Gamma_{t}}{\left(\Gamma_{S M}\right)_{t}}, \Gamma_{t}$ is the total decay width of the t-quark, taking into account the anomalous couplings and all decay modes, $\left(\Gamma_{S M}\right)_{t}$ is the SM total decay width of the t-quark, $\frac{d \Gamma_{t \rightarrow b v e^{+}}}{d \epsilon \cdot d \cos \theta \cdot d \phi}$ is the differential partial width of the polarized t-quark decay, $\epsilon=2 E_{e^{+}} / m_{t}, \epsilon_{\max }=1, \epsilon_{\min }=r^{2}, r=m_{W} / m_{t}, \theta$ and $\phi$ - orientation angles of the positron with respect to direction of the d-quark momentum.

The first term of the formula (3) reproduces shapes of the distributions as follows from the formula for the differential width, whereas the second, third and fourth terms give additional contributions. 


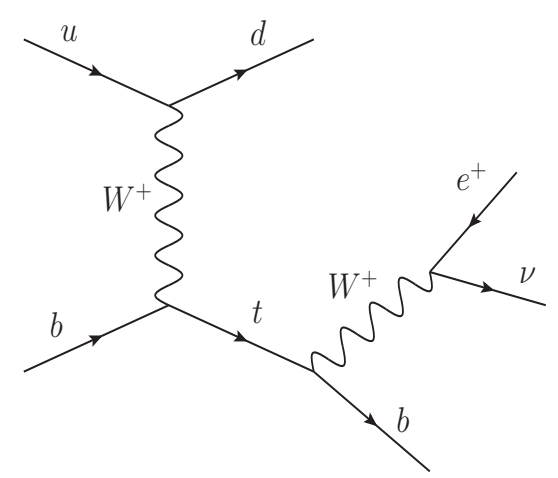

Figure 1. t-channel single top quark production and its leptonic decay processes.

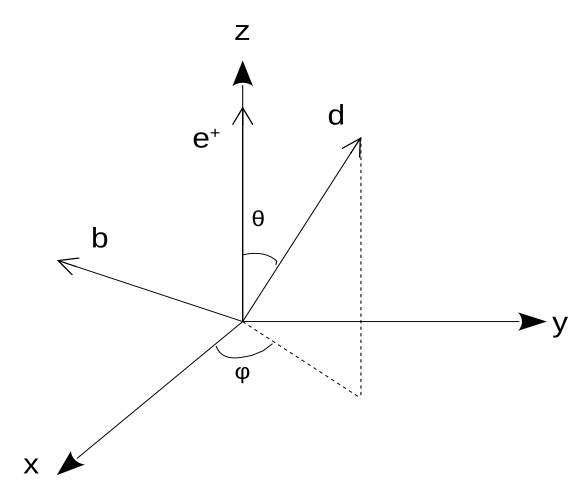

Figure 2. Top quark spin quantization axis in the top rest frame is chosen as the direction of d-quark momentum for the t-channel (outgoing light jet).

To reconstruct the $\phi$ angle in numerical Monte-Carlo simulation one can use the following expression:

$$
\phi=\arccos \left(\frac{\cos \theta_{b s}-\cos \theta_{b e} \cdot \cos \theta}{\sin \theta_{b e} \cdot \sin \theta}\right)
$$

Despite the fact that spin correlations in presence of anomalous couplings have been studied in many papers, a comparative analysis of only one-dimensional distributions corresponding to various anomalous contributions was carried out. In this case, differences may not be revealed, since a significant part of the physical information disappears when integrated over the remaining variables. We propose to compare multidimensional distributions, which are surfaces or volume regions in the space of variables. We show that in this case, the differences between the various anomalous contributions will be more significant.

\section{Top production and decay, numerical illustration.}

To verify this statement, we present the following numerical illustration for a few simple scenarios when we alternately set one of the anomalous couplings to a non-zero value. Then we show surfaces for the same kinematic variables for differential cross sections as follows from the formula (3) after integration with the parton distribution functions ${ }^{1}$ at $14 \mathrm{TeV}$ energy at the LHC collider. Note that the anomalous couplings are included both in the top quark production and decay process. Values for anomalous couplings are taken according to the latest experimental upper limits [15].

As the first scenario, we consider the case when the left vector anomalous coupling $\left(\operatorname{Re} f_{L V}-V_{t b}\right)$ is not equal to 0 , and the remaining anomalous couplings are equal to 0 . In this case, the last two terms of the expression (3) are zero, and the first two terms reproduce the same shapes of the surfaces as that for the Standard Model with $100 \%$ spin correlation behaviour $(1+\cos \theta)$, the maximum of the energy distribution at $E_{e^{+}}=m_{t} / 4 \approx 43 \mathrm{GeV}$ and no dependence on $\phi$ angle. (Fig. 3 upper) shows plots of the normalized double-differential cross sections corresponding to this scenario.

\footnotetext{
${ }^{1}$ The CTEQ61 PDF are used for definiteness. We have checked that various sets of PDF do not make an influence on shapes of the surfaces.
} 
For the second scenario, $R e f_{R V}$ is set to be non-zero while the remaining anomalous couplings are taken to be zero. Unlike the previous one, in this scenario, all terms of the formula (3) have different behaviour with respect to kinematic variables and give contributions to two-dimensional surfaces shown in (Fig. 3 middle). The first term (3) depends on used variables $\epsilon, \cos \theta$, and $\phi$ angle and reproduces the same shapes as those from the differential top width. The second term in (3) gives the shapes as for the SM slightly affecting the common shapes. The third term are zero. The fourth term does not depend of the $\phi$ angle but being proportional to the $\left|f_{R V}\right|^{2}$ coupling changes the overall shapes significantly. Since this term is proportional to $\cos \theta$, its influence is most noticeable in $\cos \theta$ distributions (Fig. 3 middle left and right).

For the third scenario, we set $\operatorname{Re} f_{L T}$ not equal to 0 , and the remaining anomalous couplings are equal to 0 . In this case, the last two terms of the expression (3) are zero and there is no dependence on the angle $\phi$ in the first and second terms. The dependence of both terms on $\cos \theta$ is the same as for $\mathrm{SM}$, but the energy distributions are different. The second term of the formula (3) slightly deviates shapes of differential cross sections (Fig. 3 down left and central) from the corresponding shapes for the differential width.

For the fourth scenario, we set $R e f_{R T}$ non-zero and positive, and the remaining anomalous couplings are equal to 0 . For this scenario, the formula for the differential cross section (3) is the most complex of all the listed scenarios. The first part of formula (3) contains quadratic anomalous terms, as well as the leading linear interference term, which mainly determines the shape of the differential cross sections. The second, third and fourth terms of the formula (3) contain quadratic anomalous terms, which only slightly affect the shape of differential cross sections (Fig. 4 upper).

For the fifth scenario, $\operatorname{Re} f_{R T}$ is set to be non-zero and negative, and the remaining anomalous couplings are equal to 0 . The overall picture is almost similar to the previous case with the only difference that deviations from the prediction of the Standard Model have the opposite sign (Fig. 4 middle).

It is clearly seen that in all the scenarios listed above, except for the second one, the shape of the differential cross section is determined mainly by the differential width of the t-quark decay. But in the case of the scenario $\left(\operatorname{Re} f_{R V} \neq 0\right)$, the second and fourth terms of the formula (3) significantly change the shape of the differential cross sections.

In the previous examples, we considered cases when only $\phi$-even terms of formula (3) are involved. To use the remaining terms, proportional to $\sin \phi$, we must consider a scenario with non-zero anomalous imaginary couplings. It should be noted that in the above simulation, we had in mind that the $\phi$ angle is restored by the formula (4). With this approach, events corresponding to the angles $\phi$ and $(2 \pi-\phi)$ are counted as events with the same $\phi$. Therefore, integration of the differential cross section (3) over the (4) from 0 to $\pi$ is equivalent to integration over the angle $\phi$ from 0 to $2 \pi$. This made sense in the considered cases where the $\phi$-even terms of formula (3) were involved. In this case, the analysis was simplified without loss of information. But not in the case of the scenarios when terms proportional to $\sin \phi$ are involved. To reflect the contribution of such terms, it is necessary to carefully separate the events corresponding to the angle $\phi$ in the range from 0 to $\pi$, and the events corresponding to $\phi$ ranging from $\pi$ to $2 \pi$ by using triple-product $T=\left(\mathbf{p}_{e^{+}} \times \mathbf{p}_{b}\right) \cdot \mathbf{s}$. If $T>0: \phi \in(0$, $\pi)$. If $T<0: \phi \in(\pi, 2 \pi)$.

For illustration with imaginary couplings, we draw plots of double-differential cross sections (Fig.4, down) for the scenario when $\operatorname{Im} f_{R T}$ are not equal to 0, and the remaining anomalous couplings are equal to 0 . For a correct comparison with the case of real coupling, we set the value of imaginary coupling $\operatorname{Im} f_{R T}=-0.048$ similar to the corresponding real one. (Fig.4, down) shows $\frac{d\left(\sigma-\sigma_{S M}\right)}{d \epsilon \cdot d \cos \theta}$ corresponding to $\phi$ ranging from 0 to $\pi$, while $\frac{d\left(\sigma-\sigma_{S M}\right)}{d \epsilon \cdot d \phi}$ and $\frac{d\left(\sigma-\sigma_{S M}\right)}{d \cos \theta \cdot d \phi}$ corresponding to $\phi$ ranging from 0 to $2 \pi$. It can be seen that deviation from the prediction of the Standard Model due to anomalous 

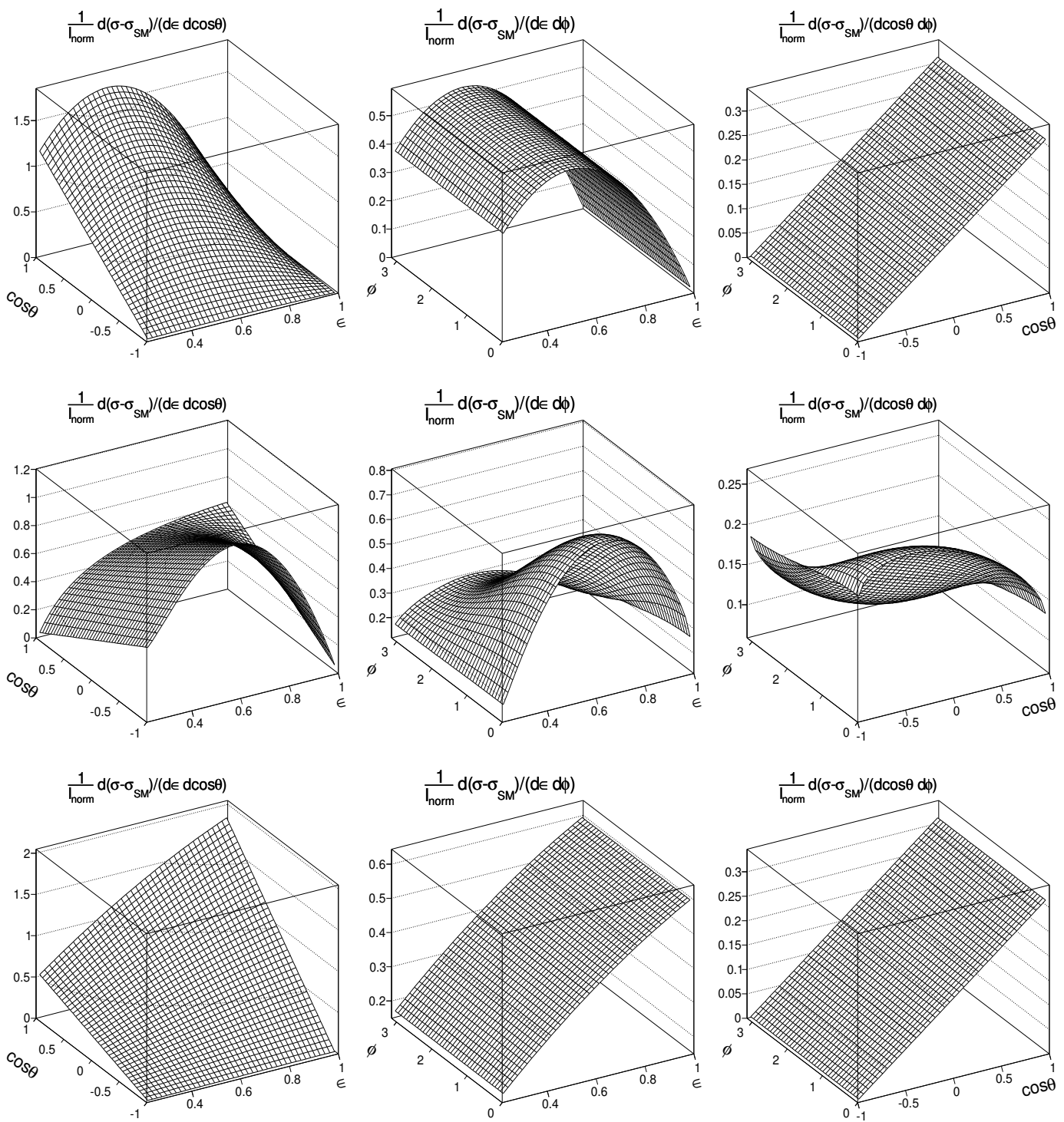

Figure 3. The figures show plots of the normalized double-differential cross sections: $\frac{d\left(\sigma-\sigma_{S M}\right)}{d \epsilon \cdot d \cos \theta}, \frac{d\left(\sigma-\sigma_{S M}\right)}{d \epsilon \cdot d \phi}$ and $\frac{d\left(\sigma-\sigma_{S M}\right)}{d \cos \theta \cdot d \phi}$ built from formula (3). The upper figures show plots corresponding to scenario $\operatorname{Re} f_{L V}=1.03$, the middle figures - to scenario $\operatorname{Re} f_{R V}=0.16$, the lower figures - to scenario $\operatorname{Re} f_{L T}=0.057$. 

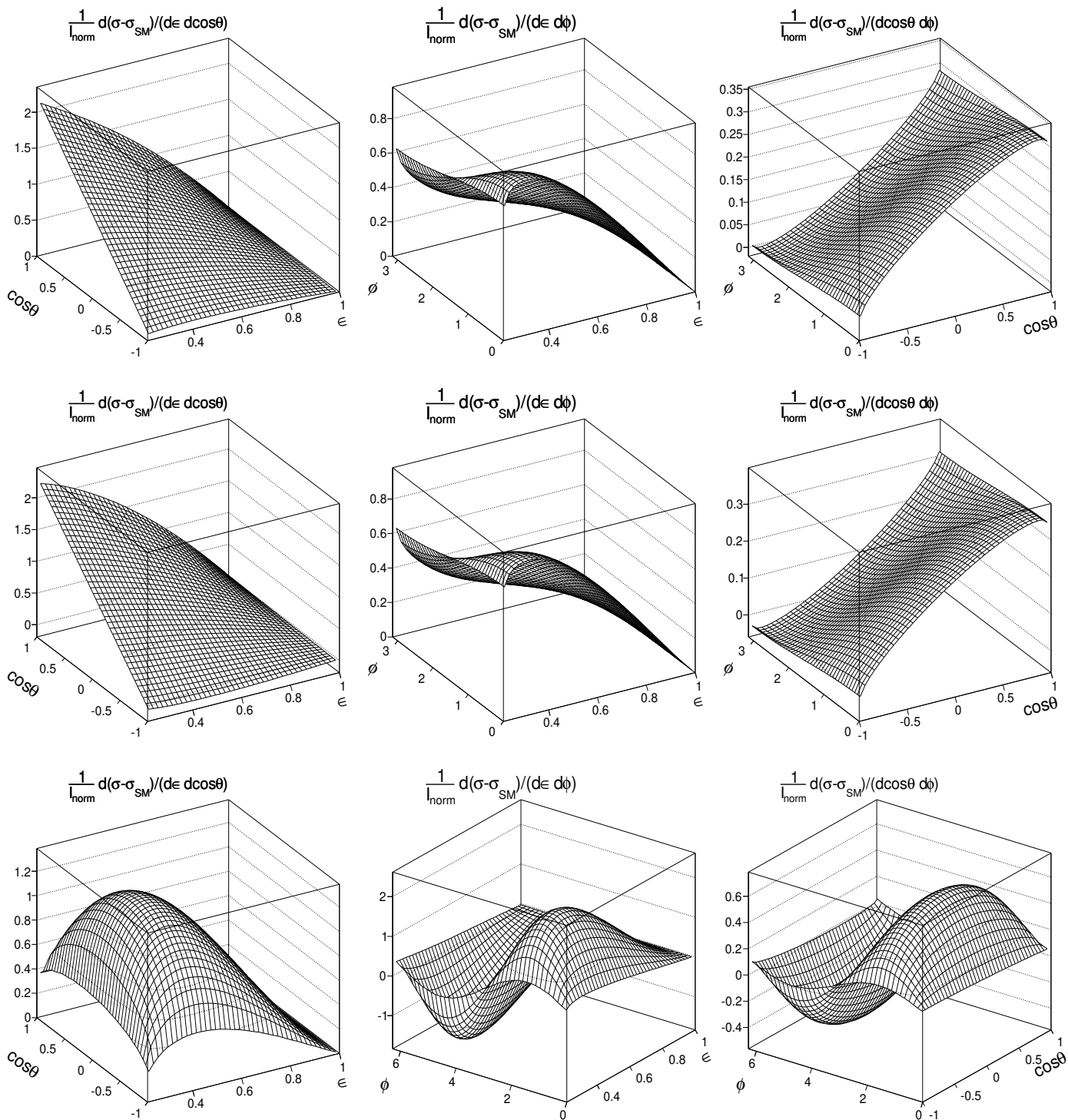

Figure 4. The figures show plots of the normalized double-differential cross sections: $\frac{d\left(\sigma-\sigma_{S M}\right)}{d \epsilon \cdot d \cos \theta}, \frac{d\left(\sigma-\sigma_{S M}\right)}{d \epsilon \cdot d \phi}$ and $\frac{d\left(\sigma-\sigma_{S M}\right)}{d \cos \theta \cdot d \phi}$ built from formula (3). The upper figures show plots corresponding to scenario $R e f_{R T}=0.048$, the middle figures - to scenario $R e f_{R T}=-0.048$, the lower figures - to scenario $\operatorname{Im} f_{R T}=-0.048$. 


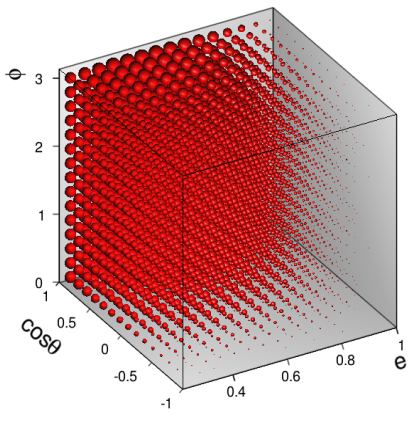

$$
R e f_{L V}=1.03
$$

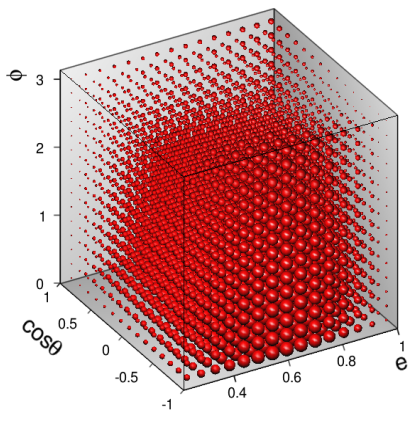

$R e f_{R V}=0.16$

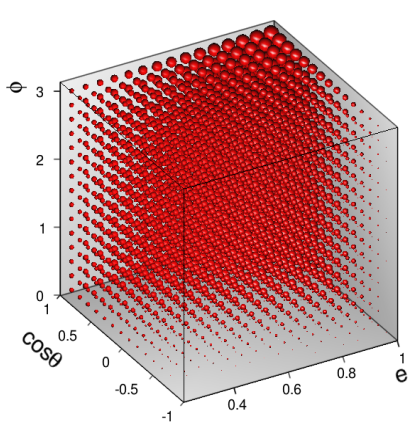

$R e f_{L T}=0.057$
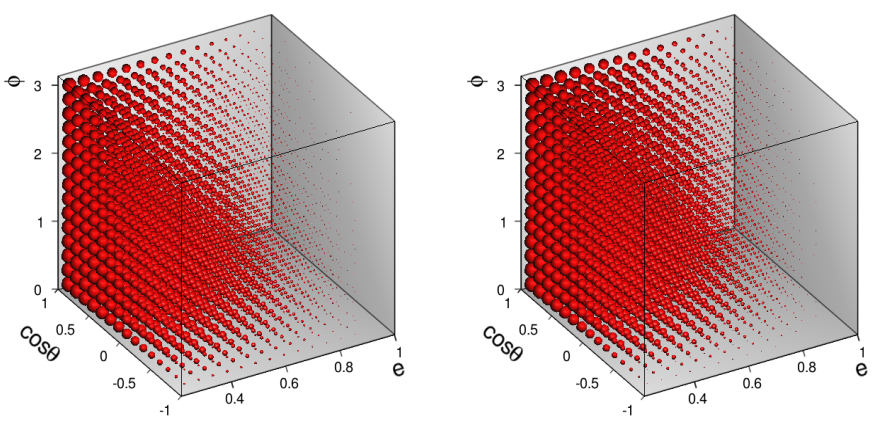

$\operatorname{Re} f_{R T}=0.048$

$$
R e f_{R T}=-0.048
$$
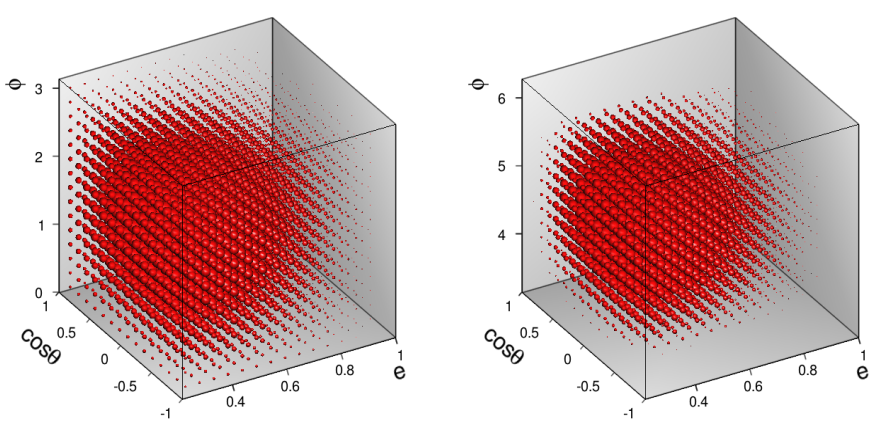

$\operatorname{Im} f_{R T}=-0.048$

Figure 5. Plots of probability density $\frac{d\left(\sigma-\sigma_{S M}\right)}{d \epsilon \cdot d \cos \theta \cdot d \phi}$ in $3 \mathrm{D}$ space $(\epsilon, \cos \theta, \phi)$ for the scenarios: $\operatorname{Re} f_{L V}=1.03$; $\operatorname{Re} f_{R V}=$ 0.16; $\operatorname{Re} f_{L T}=0.057 ; \operatorname{Re} f_{R T}=0.048 ; \operatorname{Ref}_{R T}=-0.048$ and $\operatorname{Im} f_{R T}=-0.048$. 
contributions $\left(\sigma-\sigma_{S M}\right)$ on the intervals of the angle $\phi:(0, \pi)$ and $(\pi, 2 \pi)$ differ in sign for this scenario. You can also notice a slight difference in the absolute values of the deviations from the SM, corresponding to different intervals of $\phi$ (Fig.4, down central and right). This is due to the fact that the linear anomalous term from first part of (3) is an odd function of $\phi$ and, when changing the interval of $\phi$, it changes sign, while the other quadratic anomalous terms from (3) are even and do not change. Therefore, the total sum of even and odd terms will differ at different intervals. But since the linear term dominates, and the contribution of quadratic terms is substantially suppressed, this difference is small.

As a main conclusion, it can be noted that the corresponding shapes of the surfaces in the coordinate space $(\epsilon, \cos \theta)$ and $(\epsilon, \phi)$ are very different for various scenarios. The most spectacular differences can be observed in coordinate space $(\epsilon, \cos \theta)$. This allows one to separate all scenarios from each other.

In addition to the two-dimensional distributions, we draw plots showing the probability density $\frac{d\left(\sigma-\sigma_{S M}\right)}{d \epsilon \cdot d \cos \theta \cdot d \phi}$ in the 3D space $(\epsilon, \cos \theta, \phi)$ for the cases listed (Fig. 5). The size of each cell on the figures is proportional to the probability density. One can see that the areas of maximum density for different scenarios are located in different places of the $3 \mathrm{D}$ cube $(\epsilon, \cos \theta, \phi)$. Although the three-dimensional areas are almost the same for cases $\left(R e f_{R T}=0.048\right)$ and $\left(R e f_{R T}=-0.048\right)$, the corresponding deviations from the Standard Model due to anomalous contributions for these scenarios have the opposite signs. For the last scenario, we plot the probability density corresponding to the different $\phi$ intervals: $(0, \pi)$ and $(\pi, 2 \pi)$ in different figures (Fig.5, down left and right) and their respective contributions have a different sign.

It should be noted that various combinations of real and imaginary couplings belonging to the terms of formula (3) that are odd with respect to the $\phi$ angle can manifest themselves in the form of asymmetries of differential cross sections at different intervals of $\phi:(0, \pi)$ and $(\pi, 2 \pi)$. The shapes of such distributions differ significantly from the corresponding distributions for cases of pure real couplings, which will allow us to experimentally detect and identify imaginary couplings.

\section{Conclusions}

Using the obtained analytical expressions, we plot two-dimensional distributions $\frac{d\left(\sigma-\sigma_{S M}\right)}{d \epsilon \cdot d \cos \theta}, \frac{d\left(\sigma-\sigma_{S M}\right)}{d \epsilon \cdot d \phi}$ and $\frac{d\left(\sigma-\sigma_{S M}\right)}{d \cos \theta \cdot d \phi}$ corresponding to different anomalous scenarios The most noticeable differences appear in the shape of the surfaces of two-dimensional distributions, where one of the variables is the energy of a charged lepton, and the other is one of the t-quark spin orientation angles. Also we draw plots showing the probability density $\frac{d\left(\sigma-\sigma_{S M}\right)}{d \epsilon \cdot d \cos \theta \cdot d \phi}$ in the 3D space $(\epsilon, \cos \theta, \phi)$ for the listed scenarios and show that the areas of maximum density for different scenarios are located in different places of the $3 \mathrm{D}$ cube $(\epsilon, \cos \theta, \phi)$.

\section{Acknowledgements}

The work was supported by grant 16-12-10280 of Russian Science Foundation. The authors are grateful to L. Dudko and Y. Kurihara for useful discussions and critical remarks.

\section{References}

[1] F. Déliot, N. Hadley, S. Parke and T. Schwarz, "Properties of the Top Quark," Ann. Rev. Nucl. Part. Sci. 64, 363 (2014). 
[2] E. Boos, O. Brandt, D. Denisov, S. Denisov and P. Grannis, "The top quark (20 years after its discovery)," Phys. Usp. 58, no. 12, 1133 (2015) [Usp. Fiz. Nauk 185, no. 12, 1241 (2015)]

[3] C. E. Gerber and C. Vellidis, "Review of Tevatron results: Top quark physics," Int. J. Mod. Phys. A 30, no. 06, 1541005 (2015).

[4] W. Bernreuther and P. Uwer, "Top-quark physics at colliders," Nucl. Part. Phys. Proc. 261-262, 414 (2015).

[5] M. Cristinziani and M. Mulders, “Top-quark physics at the Large Hadron Collider," J. Phys. G 44 (2017) no.6, 063001

[6] U. Husemann, “Top-Quark Physics: Status and Prospects,” Prog. Part. Nucl. Phys. 95 (2017) 48

[7] W. Buchmuller, D. Wyler, "Effective Lagrangian analysis of new interactins and flavour conservation," Nucl.Phys. B268, 621 (1986)

[8] B. Grzadkowski, M. Iskrzynski, M. Misiak and J. Rosiek, "Dimension-Six Terms in the Standard Model Lagrangian,” JHEP 1010, 085 (2010)

[9] J. A. Aguilar-Saavedra et al., "Interpreting top-quark LHC measurements in the standard-model effective field theory," arXiv:1802.07237 [hep-ph].

[10] K. Whisnant, J. M. Yang, B. L. Young and X. Zhang, "Dimension-six CP conserving operators of the third family quarks and their effects on collider observables," Phys. Rev. D 56, 467 (1997)

[11] E. Boos, M. Dubinin, M. Sachwitz and H. J. Schreiber, "Probe of the W t b coupling in t anti-t pair production at linear colliders," Eur. Phys. J. C 16 (2000) 269

[12] J. A. Aguilar-Saavedra, "Single top quark production at LHC with anomalous Wtb couplings," Nucl. Phys. B 804, 160 (2008)

[13] J. L. Birman, F. Déliot, M. C. N. Fiolhais, A. Onofre and C. M. Pease, "New limits on anomalous contributions to the Wtb vertex," Phys. Rev. D 93, no. 11, 113021 (2016)

[14] G. L. Kane, G. A. Ladinsky and C. P. Yuan, "Using the Top Quark for Testing Standard Model Polarization and CP Predictions," Phys. Rev. D 45, 124 (1992).

[15] V. Khachatryan et al. [CMS Collaboration], "Search for anomalous Wtb couplings and flavourchanging neutral currents in t-channel single top quark production in pp collisions at $\sqrt{s}=7$ and 8 TeV," JHEP 1702, 028 (2017)

[16] M. Jezabek and J. H. Kuhn, "V-A tests through leptons from polarized top quarks," Phys. Lett. B 329, 317 (1994)

[17] M. Jezabek, “Top quark physics,” Nucl. Phys. Proc. Suppl. 37B, no. 2, 197 (1994)

[18] G. Mahlon and S. J. Parke, "Improved spin basis for angular correlation studies in single top quark production at the Tevatron," Phys. Rev. D 55, 7249 (1997)

[19] G. Mahlon and S. J. Parke, "Single top quark production at the LHC: Understanding spin," Phys. Lett. B 476, 323 (2000)

[20] E. Boos and V. Bunichev, "Symbolic expressions for fully differential single top quark production cross section and decay width of polarized top quark in presence of anomalous Wtb couplings," arXiv:1910.00710 [hep-ph]. 